\title{
Fauna de répteis dos remanescentes florestais e cultivos agrícolas da Fazenda Cambuhy, municípios de Matão, Nova Europa e Tabatinga, região Noroeste do estado de São Paulo
}

\author{
Ana Bárbara Barros ${ }^{1}$ \\ Frederico de Alcântara Menezes ${ }^{2}$ \\ José Ricardo Falconi ${ }^{3}$ \\ João Gabriel Ribeiro Giovanelli ${ }^{4 *}$ \\ ${ }^{1}$ Instituto Federal Sul de Minas, Campus Inconfidentes, Inconfidentes - MG, Brasil \\ ${ }^{2}$ Universidade Federal de Viçosa, Viçosa - MG, Brasil \\ ${ }^{3}$ Supervisão de Parques Florestais, Cambuhy Agrícola Ltda., Matão - SP, Brasil \\ ${ }^{4}$ Seleção Natural - Inovação em Projetos Ambientais, Piracicaba - SP, Brasil \\ Departamento de Zoologia, Instituto de Biociências, Universidade Estadual Paulista (UNESP) \\ Avenida 24 A, 1515, Bela Vista, CEP 13506-900, Rio Claro - SP, Brasil \\ * Autor para correspondência \\ jgiovanelli@gmail.com
}

Submetido em 15/06/2016

Aceito para publicação em 10/01/2017

\section{Resumo}

A região Noroeste é a mais desmatada e com menor concentração de Unidades de Conservação do estado de São Paulo. Apesar dos avanços científicos recentes, ainda existem lacunas de conhecimento de herpetofauna. O presente estudo teve como objetivo fornecer informações sobre riqueza, abundância e uso do habitat dos répteis da Fazenda Cambuhy, que abrange os municípios de Matão, Nova Europa e Tabatinga. As amostragens ocorreram entre os anos de 2013 a 2015, utilizando-se quatro metodologias: armadilhas de interceptação e queda, procura visual limitada por tempo, procura em estradas e encontros ocasionais. Foram incluídos também os registros disponíveis em coleções científicas e na literatura. Foram registradas 46 espécies de répteis pertencentes aos grupos Squamata e Crocodylia. As espécies mais frequentes foram Salvator merianae $(\mathrm{N}=55)$, espécie mais abundante em todos os ambientes amostrados, Notomabuya frenata $(\mathrm{N}=14)$, amostrada somente nas florestas e Ameiva ameiva $(\mathrm{N}=4)$, registrada nos ambientes antrópicos e florestais. A composição de espécies registrada reflete o posicionamento da área de estudo, apresentando a maioria das espécies associadas aos biomas Cerrado e Mata Atlântica. A riqueza de répteis da Fazenda Cambuhy representa 21\% da riqueza de répteis do estado, sendo de grande significância para conservação da herpetofauna da região Noroeste do estado de São Paulo.

Palavras-chave: Cerrado; Herpetofauna; Inventário; Mata Atlântica; Matriz; Riqueza

\section{Abstract}

Reptiles of forest remnants and agricultural fields on Cambuhy Farm, in the municipalities of Matão, Nova Europa, and Tabatinga, northwestern São Paulo, Brazil. In São Paulo State, the northwestern region 
is the most deforested and has the lowest concentration of protected areas. Despite recent scientific advances, there are still gaps in the herpetological knowledge for this region. The objective of the present study was to provide information about richness, abundance and habitat use of reptiles on Cambuhy Farm, which is in the municipalities of Matão, Nova Europa, and Tabatinga. Sampling occurred from 2013 to 2015, and used four methods: pitfall traps, visual search limited by time, visual search on roads and occasional records. The study also included records from the literature and biological collections. We recorded 46 species of reptiles belonging to the Squamata and Crocodylia groups. The most common species were Salvator merianae $(\mathrm{N}=55)$, the most abundant species in all study sites, Notomabuya frenata $(\mathrm{N}=14)$, sampled only in the forests, and Ameiva ameiva $(\mathrm{N}=4)$ that was recorded in anthropic and forest environments. The species composition recorded reflects the location of the study area, with the majority of species associated with the Cerrado and Atlantic Forest biomes. The richness of reptiles on Cambuhy Farm represents $21 \%$ of the richness in São Paulo, and is of great significance for the conservation of the herpetofauna in the northwestern region of the state.

Key words: Atlantic Forest; Cerrado; Herpetofauna; Inventory; Matrix; Richness

\section{Introdução}

Atualmente 212 espécies de répteis são conhecidas para o estado de São Paulo (ZAHER et al., 2011), o que representa $27 \%$ da riqueza de espécies desse grupo no país (SBH, 2016). Após o advento do Programa Biota - FAPESP, que apoiou projetos de prospecção da biodiversidade bem como a sistematização desta informação em um banco de dados de amplo acesso (SinBiota, http://sinbiota.cria.org.br/), houve um aumento no conhecimento da herpetofauna, principalmente no interior do estado de São Paulo (e.g. SAWAYA et al., 2008; ARAÚJO et al., 2010; ARAÚJO; ALMEIDASANTOS, 2011; PEREIRA; SAWAYA, 2012).

Apesar desses avanços, ainda existem diversas lacunas de conhecimento em zonas de transição entre os biomas Cerrado e Mata Atlântica (ZAHER et al., 2011), principalmente no Noroeste do estado de São Paulo. Nessa região os remanescentes de vegetação nativa, compostos essencialmente por Floresta Estacional Semidecidual e Cerrado, foram gradativamente substituídos por pastagens, culturas agrícolas ou áreas urbanas. Atualmente, essa formação vegetal de transição entre Mata Atlântica e Cerrado restringe-se a apenas 9\% de sua cobertura original (NECCHI-JUNIOR, 2012). O estado de conservação atual coloca a região como a mais desmatada e fragmentada do estado e com menor concentração de Unidades de Conservação (KRONKA et al., 1993).

Estudos sobre a biologia e distribuição geográfica dos répteis podem servir de base para a elaboração de medidas efetivas de manejo e conservação, principalmente para o Cerrado no estado de São Paulo (ARAÚJO; ALMEIDA-SANTOS, 2011). Em um dos poucos estudos sobre a fauna de répteis Squamata da região noroeste do estado de São Paulo, Pereira e Sawaya (2012) mostram que esta encontra-se possivelmente depauperada, com populações em declínio visto as baixas abundâncias de muitas populações. $\mathrm{O}$ mesmo estudo conclui ainda que a perda de remanescentes florestais pode acarretar na extinção local de diversas espécies, já que $30 \%$ das espécies vivem exclusivamente nos fragmentos florestais. Nesse contexto de carência de informação e alto grau de ameaça, o presente trabalho teve como objetivo contribuir para o conhecimento da fauna de répteis da região noroeste de São Paulo. Principalmente fornecer informações sobre riqueza, abundância e uso do hábitat dos répteis nos diferentes ambientes naturais e antrópicos encontrados na região dos municípios de Matão, Nova Europa e Tabatinga, incluindo também dados sobre a fauna de répteis da Mata da Virgínia, o maior remanescente de vegetação nativa da região.

\section{Material e Métodos}

\section{Área de estudo}

Os ambientes amostrados neste estudo localizam-se no interior da Fazenda Cambuhy, uma propriedade rural de 14.083 ha, localizada na transição entre os biomas Cerrado e a Mata Atlântica, que abrange os municípios 
de Matão, Nova Europa e Tabatinga, estado de São Paulo (Figura 1). A vegetação nativa predominante na fazenda é a Floresta Estacional Semidecidual, com porções bastante restritas de Savana Florestada (Cerradão), que se encontram fragmentadas e imersas em uma matriz de uso predominantemente agrícola, existindo cultivos de laranja, seringueira e cana-de-açúcar (Figura 1).

A Fazenda Cambuhy possui posição estratégica em termos de conservação em razão de seus remanescentes florestais. As áreas destinadas à conservação dos ambientes naturais totalizam $4.383,3$ ha $(30,8 \%$ do total da fazenda). Destes remanescentes, $59,0 \%$ podem ser considerados em boas condições de conservação (estágios médio e avançado de regeneração de Floresta Estacional Semidecidual), sendo que a superfície florestal especificamente em estágio avançado contempla atualmente 415,1 ha ou $9,5 \%$ dos ambientes naturais. Neste contexto se destaca a Mata da Virgínia, com

FIGURA 1: Mapa de uso e ocupação do solo da Fazenda Cambuhy, região dos municípios de Matão, Nova Europa e Tabatinga, estado de São Paulo. Os pontos plotados nos mapas se referem a localização das Armadilhas de Interceptação e Queda (AIQ), utilizadas durante as amostragens, que foram instaladas em diferentes ambientes da fazenda.

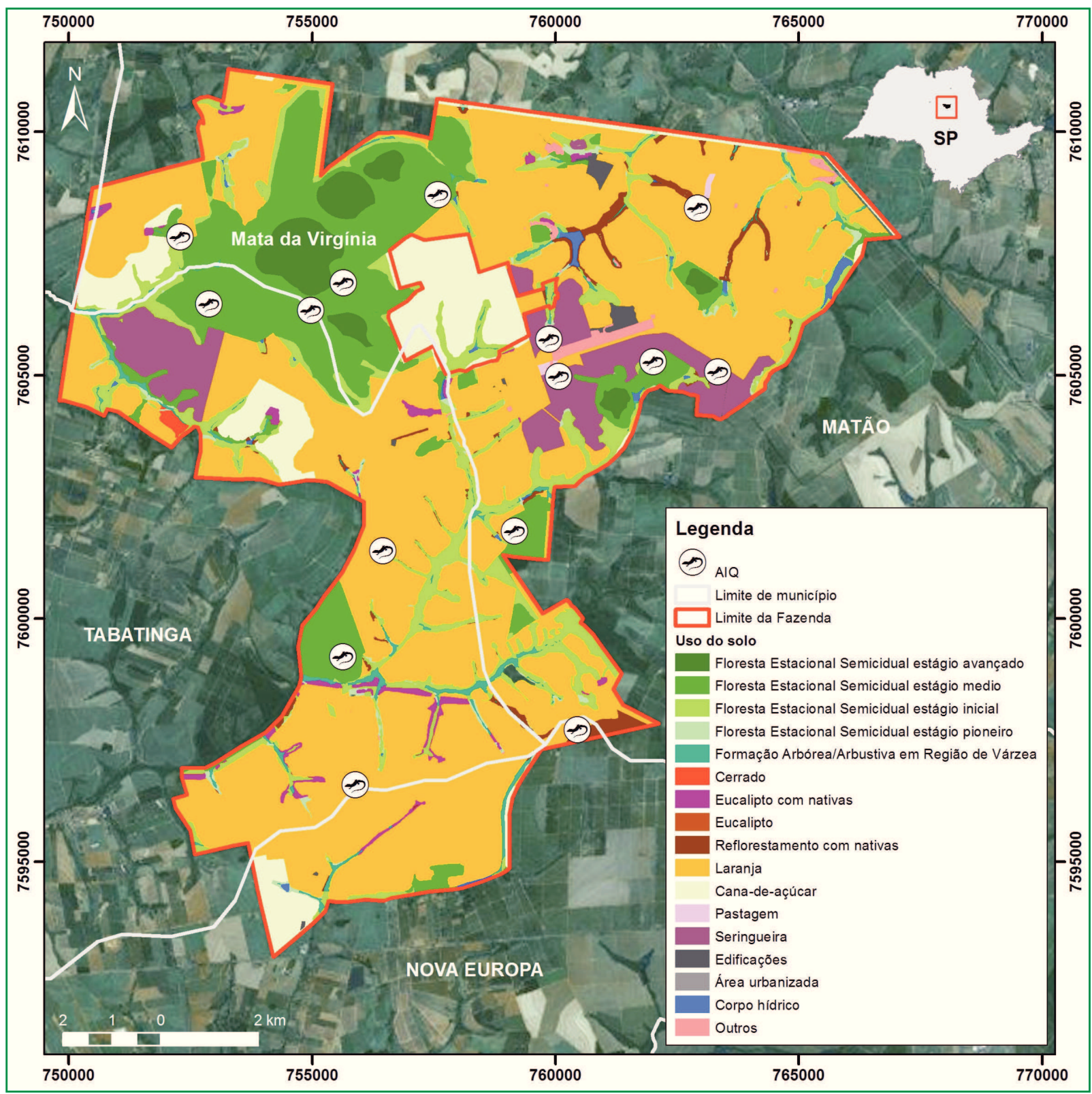


2.200 ha de Floresta Estacional Semidecidual, o maior fragmento florestal do noroeste do estado de São Paulo (Figura 1).

O clima da região é do tipo Tropical Quente e Úmido (Aw de Köppen). As temperaturas médias anuais são sempre maiores que $25^{\circ} \mathrm{C}$, com as médias dos meses mais frios (junho e julho) de cerca de $20^{\circ} \mathrm{C}$ e a dos meses mais quentes (janeiro e fevereiro) de cerca de $30^{\circ} \mathrm{C}$, com precipitação anual entre 1.100 e 1.250 mm (BARCHA; ARID, 1971; NECCHI-JUNIOR, 2012). A topografia é suave e o relevo ondulado, relativamente uniforme, com amplos e baixos espigões (ARID et al., 1970). A altitude varia de 515 a $620 \mathrm{~m}$.

\section{Amostragem dos répteis}

As amostragens de répteis em campo ocorreram na estação quente e chuvosa dos anos de 2013,
2014 e 2015. Foram utilizados quatro métodos de amostragem: 1) armadilhas de interceptação e queda (AIQ) (GREENBERG et al., 1994; CECHIN; MARTINS, 2000); 2) procura visual limitada por tempo (PVLT) (CAMPBELL; CHRISTMAN, 1982; SCOTT-JR. et al., 1989; MARTINS; OLIVEIRA, 1998); 3) procura em estradas (SAWAYA et al., 2008); e 4) encontros ocasionais.

Com relação às armadilhas de interceptação e queda (AIQ), em cada uma das localidades amostradas foi instalada uma linha de armadilhas composta por quatro baldes de $60 \mathrm{~L}$, conectados por $10 \mathrm{~m}$ de cerca-guia (com aproximadamente $60 \mathrm{~cm}$ de altura), resultando em linhas de $40 \mathrm{~m}$ de extensão. No total, foram amostradas 15 localidades nos diferentes ambientes da fazenda (Figuras 1 e 2). Foram amostrados 30 dias não consecutivos de armadilhas, que corresponderam a 1.620 dias-balde.

FIGURA 2: Armadilhas de Interceptação e Queda foram instaladas nos ambientes (a) florestais, (b) cultivos de laranja e (c) seringueira.
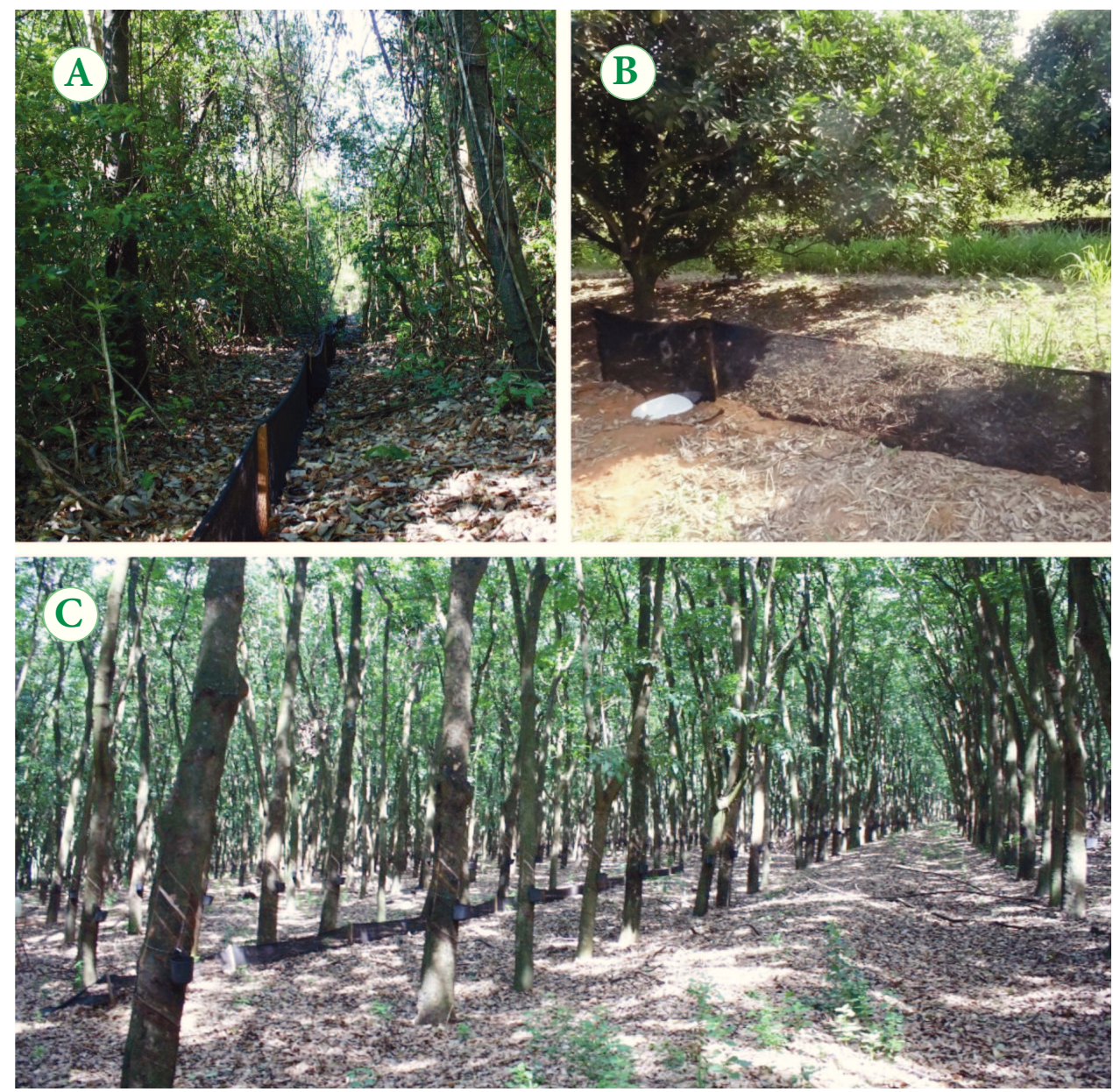
A procura visual limitada por tempo (PVLT), que consiste no deslocamento a pé, na busca por espécimes, em todos os microambientes visualmente acessíveis, foi realizada nas mesmas localidades amostradas por AIQ (Figura 1). No total, foram realizadas 75 horas-pessoa de procura visual nos diferentes ambientes da fazenda.

A procura em estradas (PE) correspondeu ao encontro de répteis avistados em estradas e carreadores (vias localizadas dentro dos cultivos agrícolas) percorridos com veículo. Foram utilizadas as estradas e carreadores percorridos para a amostragem das armadilhas de interceptação e queda. O esforço foi quantificado em $\mathrm{km}$ rodados e normalmente a velocidade do veículo foi de 20 a $40 \mathrm{~km} / \mathrm{h}$. No total foram amostrados $1.425 \mathrm{~km}$.

Os encontros ocasionais (EO) corresponderam ao encontro dos répteis vivos ou mortos durante outras atividades que não a amostragem dos demais métodos. Essa atividade foi feita principalmente pelos funcionários da fazenda, que fotografaram os indivíduos vivos que forrageavam ao redor das edificações e nas estradas, e coletavam também as carcaças de indivíduos encontrados mortos. $\mathrm{O}$ esforço amostral dos encontros ocasionais foi considerado como o número total de dias de duração do projeto, que foi de três anos, correspondendo a 1.095 dias.

A lista de espécies foi complementada também com o registro dos répteis dos municípios de Matão, Nova Europa e Tabatinga em coleções biológicas de referência e participantes do speciesLink, um sistema distribuído de informação que integra, em tempo real, dados primários de coleções científicas (SPECIESLINK, 2016).

Os animais pertencentes a grupos de difícil determinação taxonômica foram coletados e eutanasiados com Lidocaína ${ }^{\circledR}$ injetável, fixados com formol a $10 \%$ e preservados com álcool a 70\% (autorização de coleta concedida pelo Sistema de Autorização e Informação em Biodiversidade - SISBIO no 40357-4). Todos os exemplares coletados foram identificados e serão depositados no Museu de Zoologia da Universidade Estadual de Campinas.

\section{Análise dos dados}

A lista de espécies de répteis segue a nomenclatura adotada pela Sociedade Brasileira de Herpetologia (SBH, 2016). O estado de conservação das espécies foi avaliado segundo listas de espécies consideradas ameaçadas pela legislação estadual (BRESSAN et al., 2009), federal (MMA, 2014) e de âmbito global (IUCN, 2016). Informações sobre endemismo e ocorrência das espécies nas diferentes fitofisionomias do estado de São Paulo foram compiladas do trabalho de Zaher et al. (2011).

A partir dos dados primários, foram analisadas a composição, riqueza e frequência de captura de espécies de répteis. Devido ao baixo número de capturas, principalmente nos métodos sistemáticos, a frequência para os répteis amostrados pelas AIQ, PVLT, PE e EO foi estabelecida em porcentagem (e.g. FORLANI et al., 2010).

A comparação entre as taxocenoses de répteis foi conduzida através do programa Past (HAMMER et al., 2001), empregando-se o índice de similaridade de Jaccard (MAGURRAN, 2004), e o método de agrupamento utilizado foi o de UPGMA. Para comparação foram selecionados trabalhos de inventário de répteis, sendo três deles realizados no bioma Cerrado (UETANABARO et al., 2007 - Serra da Bodoquena, MS; ARAÚJO; ALMEIDA-SANTOS, 2011 - Estação Ecológica de Assis, SP; RECODER et al., 2011 - Serra Geral, TO) e três na Mata Atlântica (CICCHI et al., 2009 - Ilha Anchieta, SP; CONDEZ et al., 2009 - Tapiraí, SP; FORLANI et al., 2010 - Carlos Botelho, SP).

\section{Resultados}

\section{Composição de espécies}

Considerando-se os dados primários e secundários, no total foram registradas 46 espécies de répteis, pertencentes aos grupos Squamata e Crocodylia (Figura 3). O grupo Squamata inclui os lagartos, as serpentes e as anfisbenas (cobra-de-duas-cabeças). No caso dos lagartos, foi possível registrar sete espécies, pertencentes às famílias Teiidae (três 
espécies), Dactyloidae (uma espécie), Mabuyidae (uma espécie), Anguide (uma espécie) e Tropiduridae (uma espécie). Com relação às serpentes, foram registradas 37 espécies, pertencentes às famílias Boidae (três espécies), Dipsadidae (21 espécies), Colubridae (cinco espécies), Viperidae (cinco espécies) e Elapidae (três espécies). Somente uma espécie de anfisbena (família Amphisbaenidae) foi registrada (Tabela 1). Para os crocodilianos, foi registrada somente uma espécie (Caiman latirostris), pertencente à família Alligatoridae.

FIGURA 3: Imagens selecionadas dos répteis registrados na Fazenda Cambuhy, municípios de Matão, Nova Europa e Tabatinga, região Noroeste do estado de São Paulo: (A) Caiman latirostris, (B) Salvator merianae, (C) Ameivula ocellifera, (D) Notomabuya frenata, (E) Phalotris mertensi, (F) Erythrolamprus reginae, (G) Spilotes pullatus, (H) Erythrolamprus poecilogyrus e (I) Helicops infrataeniatus.
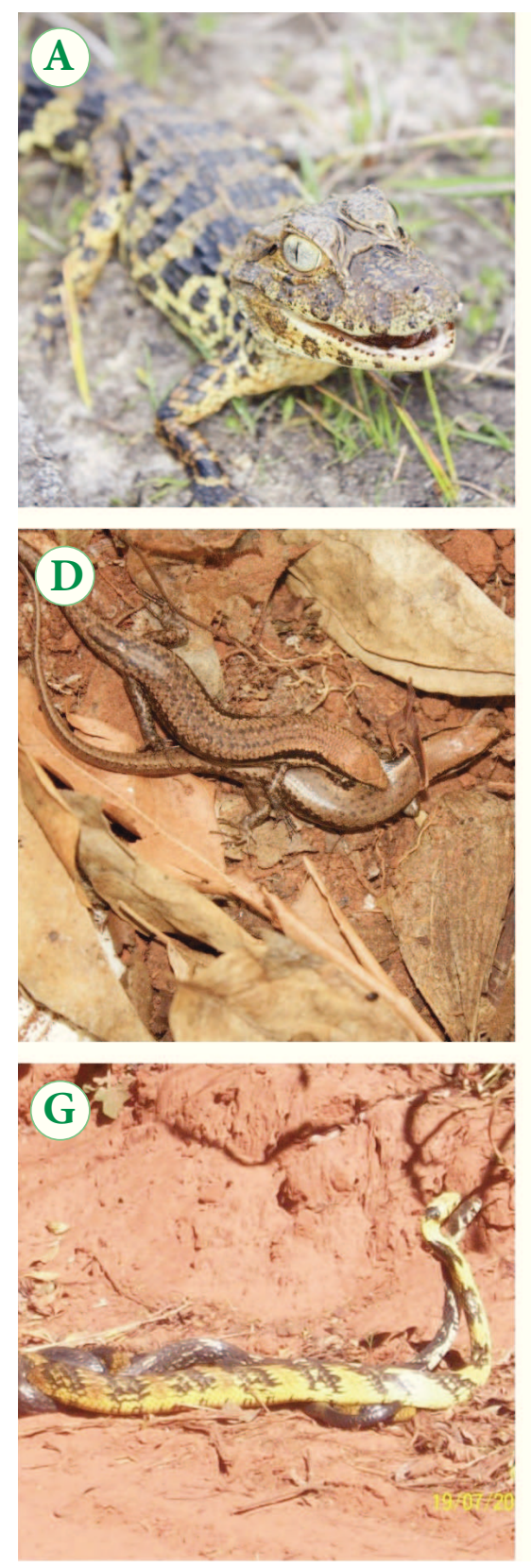
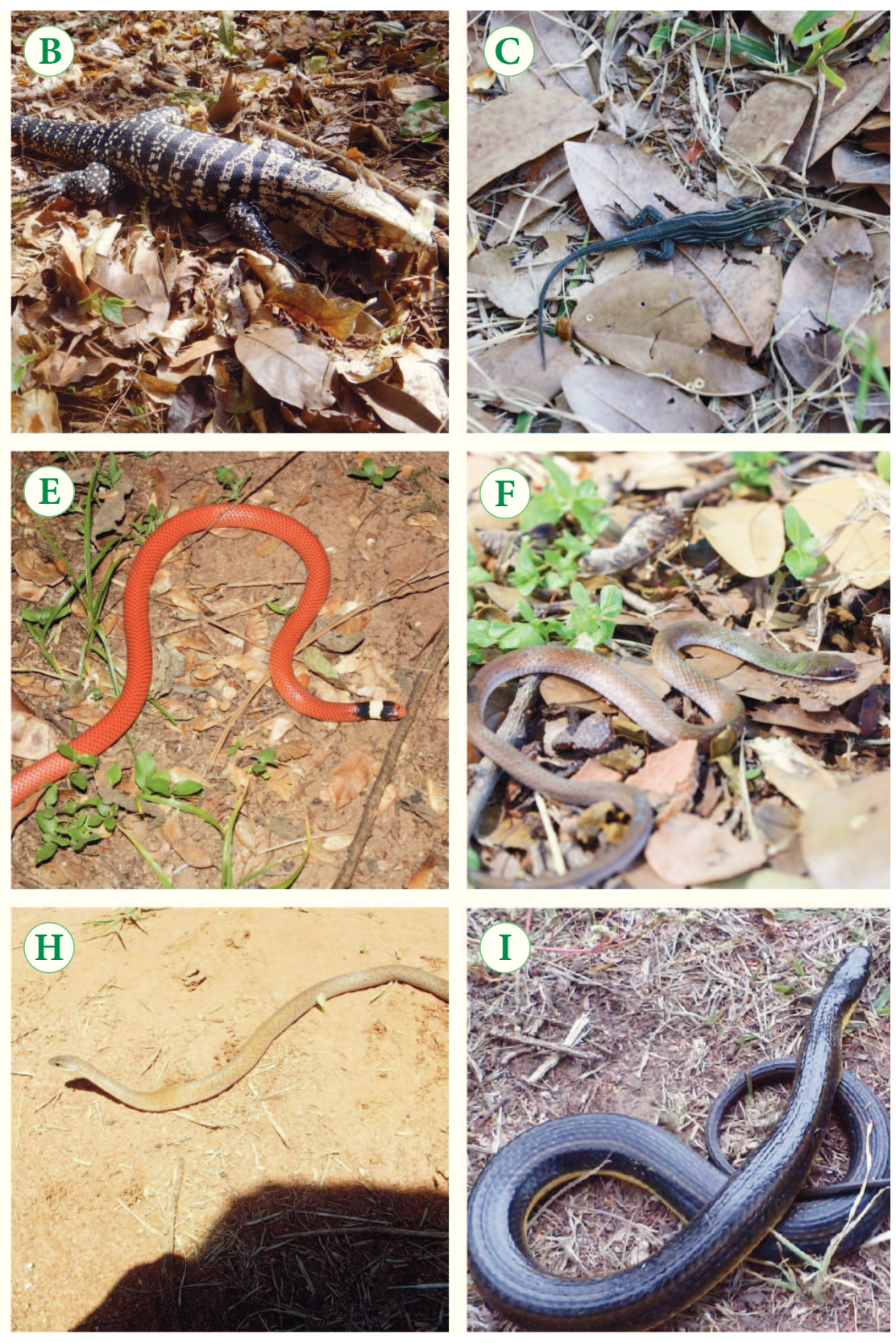
TABELA 1: Lista dos répteis da Fazenda Cambuhy, municípios de Matão, Nova Europa e Tabatinga, estado de São Paulo. Metodologias de obtenção de dados primários: AIQ (armadilhas de interceptação e queda); PVLT (procura visual limitada por tempo); PE (procura em estrada); EO (encontro ocasional). Fonte de obtenção de dados secundários: 1 (Coleção de Répteis, Pontifícia Universidade Católica do Rio Grande do Sul, PUCRS); 2 (Sistema de Informação do Programa Biota/Fapesp - SinBiota); 3 (Coleção Herpetológica “Alphonse Richard Hoge", Instituto Butantan); 4 (PEREIRA; SAWAYA, 2012). Local de Registro: A (Ambiente Antrópico); CA (Corpo d'água); F (Floresta); L (Laranja); S (Seringueira). * Espécies ameaçadas de extinção.

\begin{tabular}{|c|c|c|c|}
\hline Família/espécie & Dados primários & Dados secundários & $\begin{array}{l}\text { Local do } \\
\text { Registro }\end{array}$ \\
\hline \multicolumn{4}{|l|}{ Alligatoridae } \\
\hline Caiman latirostris (Daudin, 1801) & EO & 2 & $\mathrm{CA}$ \\
\hline \multicolumn{4}{|l|}{ Teiidae } \\
\hline Ameiva ameiva (Linnaeus, 1758) & AIQ, PE & - & $\mathrm{A}, \mathrm{F}$ \\
\hline Ameivula ocellifera (Spix, 1825) & EO & 4 & $\mathrm{~A}, \mathrm{~F}$ \\
\hline Salvator merianae (Duméril e Bibron, 1839) & $\mathrm{PE}$ & 4 & A, F, L, S \\
\hline \multicolumn{4}{|l|}{ Dactyloidae } \\
\hline Norops chrysolepis (Duméril e Bibron, 1837) & AIQ & 4 & $\mathrm{~F}$ \\
\hline \multicolumn{4}{|l|}{ Mabuyidae } \\
\hline Notomabuya frenata (Cope, 1862) & AIQ, PE & - & $\mathrm{F}$ \\
\hline \multicolumn{4}{|l|}{ Anguidae } \\
\hline Ophiodes fragilis (Raddi, 1820) & EO & - & $\mathrm{F}$ \\
\hline \multicolumn{4}{|l|}{ Tropiduridae } \\
\hline Tropidurus torquatus (Wied, 1820) & AIQ, PE, EO & - & F, L, S \\
\hline \multicolumn{4}{|l|}{ Amphisbaenidae } \\
\hline Amphisbaena alba Linnaeus, 1758 & EO & - & $\mathrm{A}$ \\
\hline \multicolumn{4}{|l|}{ Boidae } \\
\hline Boa constrictor Linnaeus, 1758 & $\mathrm{EO}$ & 3,4 & $\mathrm{~A}$ \\
\hline Epicrates cenchria (Linnaeus, 1758) & - & 3 & - \\
\hline Eunectes murinus (Linnaeus, 1758) & EO & - & $\mathrm{CA}$ \\
\hline \multicolumn{4}{|l|}{ Dipsadidae } \\
\hline Apostolepis dimidiata (Jan, 1862) & - & 3 & - \\
\hline Erythrolamprus aesculapii (Linnaeus, 1766) & - & 3 & - \\
\hline Erythrolamprus almadensis (Wagler in Spix, 1824) & - & 3 & - \\
\hline Erythrolamprus frenatus (Werner, 1909) & EO & - & $\mathrm{F}$ \\
\hline Erythrolamprus miliaris (Linnaeus, 1758) & EO & - & $\mathrm{F}$ \\
\hline Erythrolamprus poecilogyrus (Wied-Neuwied, 1825) & - & 3 & - \\
\hline Erythrolamprus reginae (Amaral, 1935) & AIQ & - & $\mathrm{F}$ \\
\hline Imantodes cenchoa (Linnaeus, 1758) & - & 3 & - \\
\hline Helicops carinicaudus (Wied, 1824) & - & 3 & - \\
\hline Helicops infrataeniatus (Jan, 1865) & EO & - & A \\
\hline Oxyrhopus petolarius (Linnaeus, 1758) & - & 3 & - \\
\hline Oxyrhopus rhombifer Duméril, Bibron \& Duméril, 1854 & - & 3 & - \\
\hline Oxyrhopus trigeminus Duméril, Bibron e Duméril, 1854 & - & 3 & - \\
\hline Phalotris mertensi (Hoge, 1955) & $\mathrm{PE}$ & 1,3 & $\mathrm{~F}$ \\
\hline Phalotris nasutus (Gomes, 1915)* & - & 3 & - \\
\hline Philodryas olfersii (Liechtenstein, 1823) & - & 3 & - \\
\hline
\end{tabular}




\section{Família/espécie}

Philodryas patagoniensis (Girard, 1858)

Pseudoboa haasi (Boettger, 1905)

Rhachidelus brazili Boulenger, 1908

Sibynomorphus mikanii (Schlegel, 1837)

Xenodon merremii (Wagler in Spix, 1824)

\section{Colubridae}

Chironius flavolineatus (Jan, 1863)

Chironius quadricarinatus (Boie, 1827)

Mastigodryas bifossatus (Raddi, 1820)

Simophis rhinostoma (Schlegel, 1837)

Spilotes pullatus (Linnaeus, 1758)

\section{Viperidae}

Bothrops alternatus Duméril, Bibron e Duméril, 1854

Bothrops itapetiningae (Boulenger, 1907)*

Bothrops jararaca (Wied, 1824)

Bothrops neuwiedi Wagler in Spix, 1824

Crotalus durissus (Laurenti, 1768)

\section{Elapidae}

Micrurus corallinus

Micrurus frontalis (Duméril, Bibron e Duméril, 1854)

Micrurus lemniscatus (Linnaeus, 1758)
Dados primários Dados secundários

Local do

Registro

- 3

$\begin{array}{ll}- & 3 \\ - & \end{array}$

- 3

EO, PVLT

- $\quad 3,4$

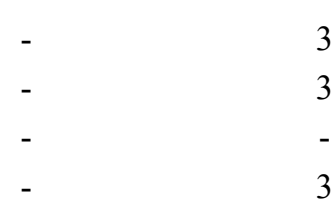

PVLT

-

$3-$

A, F

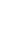

3

3

3

3

$\begin{array}{ccc}- & 3 & - \\ - & 3 & - \\ - & 3 & - \\ - & 3 & - \\ \mathrm{PE} & 3 & \mathrm{~A}, \mathrm{~F}\end{array}$

EO, PVLT

3,4

A, F

- 3

Entre as 46 espécies registradas para área de estudo, 20 foram coletadas durante os trabalhos de campo (dados primários), sendo que 11 destas não estão presentes nos dados secundários. Por outro lado, 35 espécies foram registradas através dos dados secundários, sendo que 25 espécies exclusivamente por esse método. Todos os registros inéditos obtidos a partir de dados secundários são de serpentes depositadas na Coleção Herpetológica "Alphonse Richard Hoge" do Instituto Butantan.

Entre as espécies levantadas, apenas duas espécies, registradas através de dados secundários, se encontram listadas em categorias de ameaça. A espécie Bothrops itapetiningae se encontra na categoria Quase Ameaçada (NT) de extinção na lista brasileira e na categoria Vulnerável VU na lista estadual de fauna ameaçada. Outra espécie, a Phalotris nasutus se encontra na categoria Em Perigo (EN) somente na lista estadual.

\section{Frequência dos registros e uso do habitat}

No total dos dados primários, foram registrados 102 espécimes pertencentes a 20 espécies de répteis, sendo um crocodiliano, uma anfisbênia, sete lagartos e 11 serpentes. Os mais frequentes foram o lagarto Salvator merianae $(\mathrm{N}=55 ; 54,5 \%)$, espécie mais abundante em todos os ambientes amostrados, Notomabuya frenata ( $\mathrm{N}=14 ; 13,9 \%)$, amostrada somente nas florestas e Ameiva ameiva $(\mathrm{N}=4 ; 4 \%)$, registrada nos ambientes antrópicos e nas florestas (Figura 4).

Dos 20 répteis amostrados por dados primários, 10 espécies foram registradas exclusivamente nas florestas. As espécies Caiman latirostris e Eunectes murinus foram registradas somente nas proximidades dos corpos d'água (Figura 5). O restante das espécies ocorreu em mais de um ambiente e nenhuma espécie foi registrada exclusivamente nos ambientes agrícolas. 
FIGURA 4: Frequência dos registros das espécies anotadas por dados primários na Fazenda Cambuhy, municípios de Matão, Nova Europa e Tabatinga, região Noroeste do estado de São Paulo.

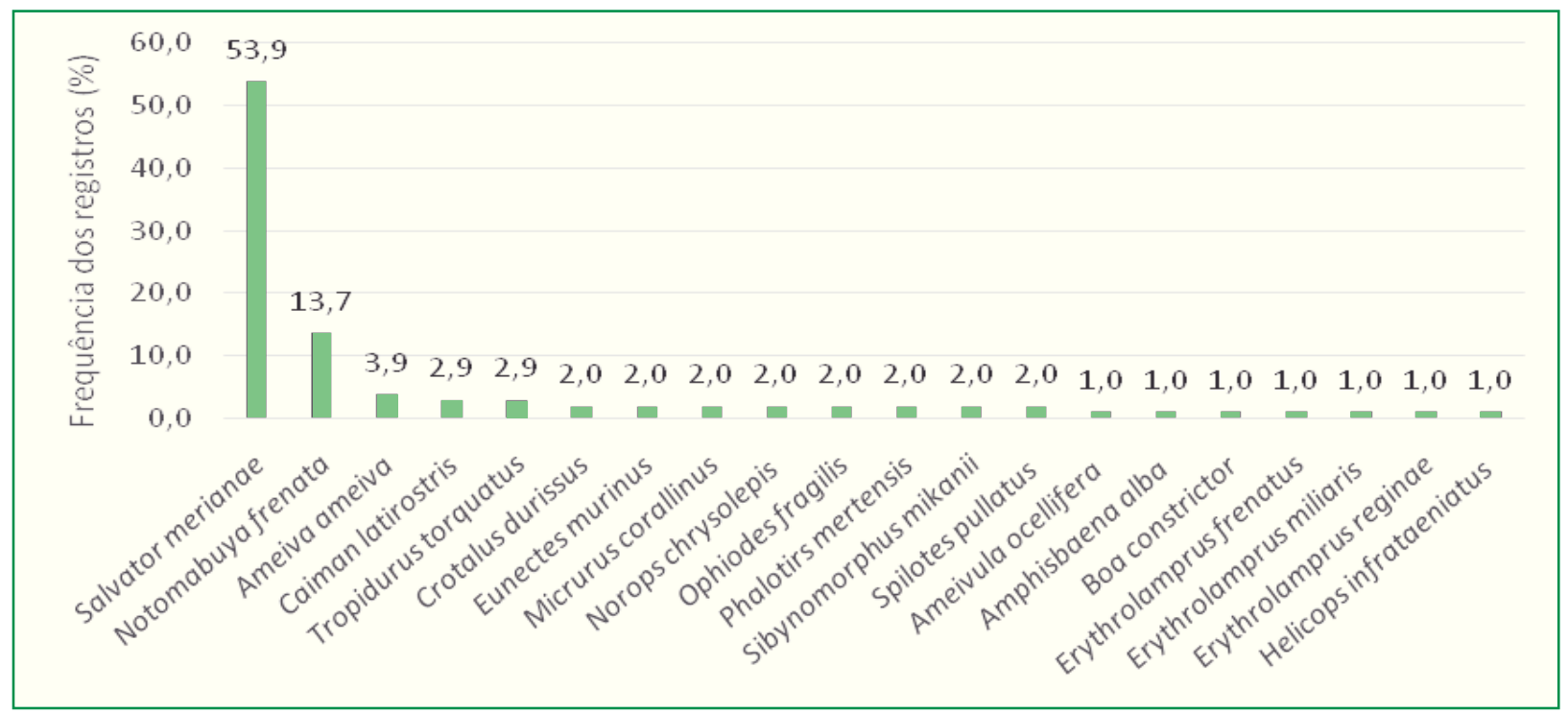

FIGURA 5: As espécies Caiman latirostris e Eunectes murinus foram registradas exclusivamente no interior e proximidades dos corpos d'água da Fazenda Cambuhy, municípios de Matão, Nova Europa e Tabatinga, região Noroeste do estado de São Paulo.

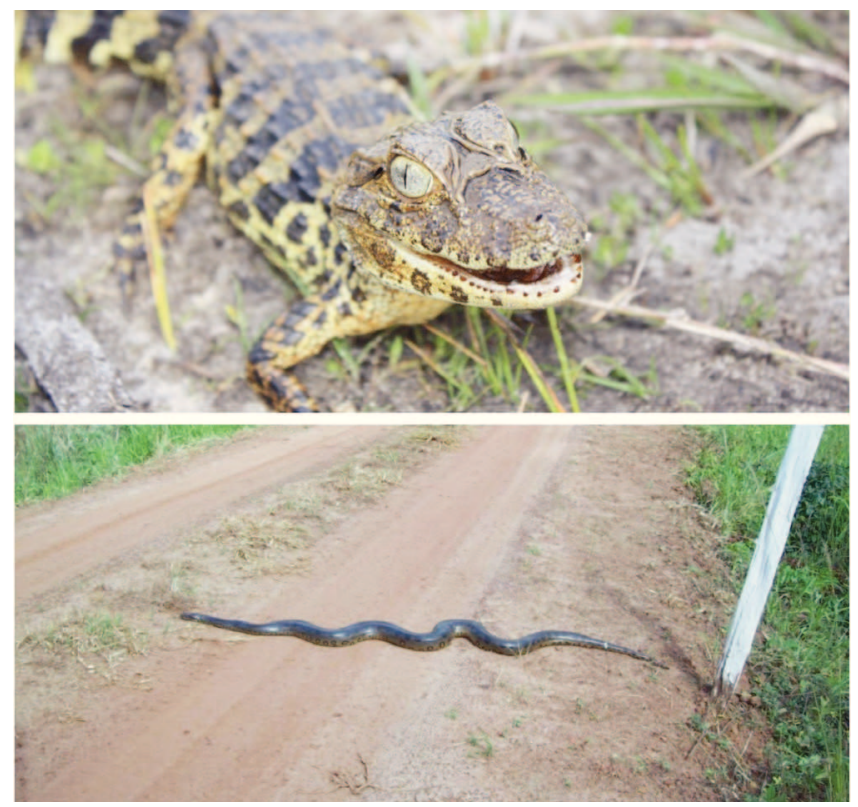

Foi possível constatar que grande parte dos espécimes registrados foi amostrada pelo método da procura de estrada ( $\mathrm{N}=72 ; 70,6 \%)$, seguido pelo das armadilhas de interceptação e queda $(\mathrm{N}=13 ; 12,7 \%)$, encontros ocasionais $(\mathrm{N}=12 ; 11,8 \%)$ e procura visual limitada por tempo $(\mathrm{N}=5 ; 4,9 \%)$.

Entre as serpentes, Eunectes murinus, Micrurus corallinus, Phalotris mertensis, Sibynomorphus mikanii e Spilotes pullatus apresentaram maior quantidade de indivíduos coletados, sendo registrados dois indivíduos para cada uma dessas espécies.

\section{Comparação com taxocenoses de outras localidades}

A análise de agrupamento (coeficiente de correlação cofenético $=0,97$ ) baseada em 164 espécies de répteis 
registradas em sete localidades, incluindo a Fazenda Cambuhy, resultou na formação de três agrupamentos principais (Figura 6): (1) um agrupamento formado pelas localidades do estado de São Paulo, apresentando uma divisão em dois subconjuntos. O primeiro (4) é formado por duas localidades inseridas no contexto do bioma da Mata Atlântica na região da Serra de Paranapiacaba, onde predomina as Florestas Ombrófilas Densas e o segundo (5) é formado por duas localidades inseridas em regiões de transição dos biomas Mata Atlântica e Cerrado, no interior do estado; (2) um agrupamento formado por duas localidades inseridas no bioma Cerrado e (3) um agrupamento formado somente pela Ilha Anchieta, que, apesar de estar inserida no bioma da Mata Atlântica do estado de São Paulo, possui características ambientais específicas de um ambiente insular (CICCHI et al., 2009).

FIGURA 6: Dendrograma de similaridade com dados de ocorrência de espécies de répteis em sete localidades: Serra da Bodoquena, MS; Estação Ecológica de Assis, SP; Serra Geral, TO; Ilha Anchieta, SP; Tapiraí, SP; Parque Estadual de Carlos Botelhos, SP. Índice de Jaccard e método de agrupamento "UPGMA" (coeficiente de correlação cofenético $=0,97$ ).

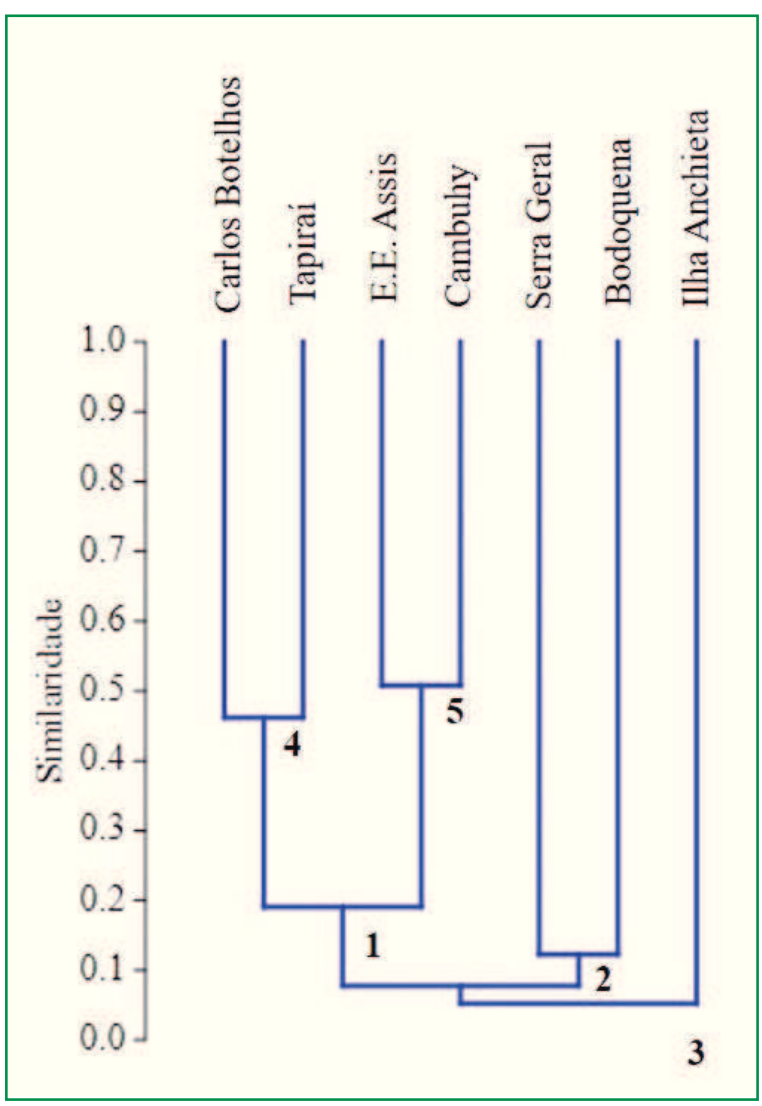

\section{Discussão}

A riqueza de espécies nos remanescentes naturais e áreas agrícolas da Fazenda Cambuhy, registradas através de dados primários e secundários, representam $21 \%$ da riqueza de répteis do estado de São Paulo (ZAHER et al., 2011). O número de espécies observado neste estudo é menor do que a riqueza inventariada para algumas Unidades de Conservação de proteção integral do estado de São Paulo (Estação Ecológica de Assis (SP): 53 espécies de répteis; Parque Estadual de Carlos Botelhos (SP): 59 espécies de répteis; Estação Ecológica de Santa Bárbara (SP): 12 espécies de lagartos). No entanto, considerando-se que a Fazenda Cambuhy possui um histórico de uso e ocupação do solo diferenciado das demais Unidades de Conservação, a riqueza de espécies inventariadas nesta localidade é significativa para a conservação da herpetofauna da região Noroeste do estado de São Paulo.

Analisando-se somente os dados primários, as espécies registradas na Fazenda Cambuhy representam $50 \%$ da riqueza de espécies inventariadas por Pereira e Sawaya (2012) em 18 fragmentos de Floresta Estacional Semidecidual e suas respectivas matrizes ao longo das regiões administrativas de São José do Rio Preto, Araçatuba, Bauru e Ribeirão Preto.

É importante salientar que os registros de Bothrops itapetiningae e Phalotris nasutus foram feitos através de dados secundários obtidos de registros históricos. São raros os registros dessas espécies no estado de São Paulo nas últimas décadas, possivelmente pela dependência de habitats campestres relativamente conservados (NOGUEIRA, 2001). Um dos últimos registros de Bothrops itapetiningae no estado foi na Estação Ecológica de Itirapina, onde ainda ocorrem diversas fisionomias de cerrado conservadas (SAWAYA et al., 2008). No caso de Phalotris nasutus, sua ocorrência atual parece estar restrita a fragmentos de cerrado no extremo norte do estado, que possivelmente representam o limite sul da área de ocorrência da espécie (FERRAREZZI, 1993).

As espécies Notomabuya frenata e Ameiva ameiva, abundantes no presente estudo, também obtiveram uma elevada frequência de ocorrência em estudos realizados 
na Estação Ecológica de Assis e na Estação Ecológica de Santa Bárbara (ARAÚJO; ALMEIDA-SANTOS, 2011; ARAÚJO et al., 2014). A espécie Salvator meriane foi a mais abundante ao longo do presente estudo. Essa espécie parece não apresentar alta frequência de registros em habitats conservados, como é o caso das Unidades de Conservação do estado de São Paulo (e.g. THOMÉ, 2006; ARAÚJO; ALMEIDA-SANTOS 2011; ARAÚJO et al., 2014). A alta frequência de registros dessa espécie pode se dar pelo fato de possuir capacidade de invadir ou colonizar, com relativo sucesso, ambientes antropizados ou com algum grau de alteração (ZAHER et al., 2011).

Conforme esperado, a comunidade de répteis da fazenda Cambuhy assemelhou-se com os estudos desenvolvidos nos biomas Mata Atlântica e Cerrado do estado de São Paulo, principalmente pelo fato de algumas espécies possuírem distribuição geográfica ampla nos biomas dessa região. O fato de a Fazenda Cambuhy se agrupar com a Estação Ecológica de Assis no subconjunto 5 (Figura 6) mostra a influência do bioma Cerrado no interior do estado de São Paulo.

De acordo com a classificação feita por Zaher et al. (2011), a composição de espécies registrada reflete o posicionamento da área de estudo entre os biomas do Cerrado e Mata Atlântica, apresentando a maioria das espécies associadas ao Cerrado e às fitofisionomias de Mata Atlântica. Além disso, todas as espécies registradas possuem ampla distribuição geográfica no estado de São Paulo.

Apesar do histórico de uso e ocupação do solo da região Noroeste do estado de São Paulo, a Fazenda Cambuhy apresenta significativa cobertura de vegetação nativa (4.383,3 ha) delimitada como Área de Preservação Permanente e Reserva Legal que pode garantir a conservação da herpetofauna local. Ações de monitoramento das espécies no futuro podem ser uma importante contribuição na geração de dados populacionais, principalmente das espécies que utilizam a matriz produtiva como área de vida.

\section{Agradecimentos}

Os autores agradecem pelas valiosas contribuições para elaboração deste estudo realizadas por Rodrigo de
Almeida Nobre, Camila Cantagallo e Andrezza Bellotto Nobre. Esta publicação é resultante do "Programa de Conservação da Biodiversidade da Mata da Virgínia, Fazenda Cambuhy, SP”, coordenado pela Seleção Natural - Inovação em Projetos Ambientais, com apoio financeiro da Cambuhy Agrícola Ltda.

\section{Referências}

ARAÚJO, C. O.; ALMEIDA-SANTOS, S. M. Herpetofauna in a cerrado remnant in the state of São Paulo, Southeastern Brazil. Biota Neotropica, Campinas, v. 11, n. 3, p. 47-62, 2011.

ARAÚJO, C. O.; CÔRREA-FILHO, D. T.; SAWAYA, R. J. Snake assemblage of Estação Ecológica de Santa Bárbara, SP: a Cerrado remnant in Southeastern Brazil. Biota Neotropica, Campinas, v. 10, n. 2, p. 235-245, 2010.

ARAÚJO, C. O.; MAFFEI, F.; CORRÊA, D. T.; MOYA, G. M.; NASCIMENTO, B. T. M.; ALMEIDA-SANTOS, S. M. Lizards from Estação Ecológica de Santa Barbára, a remnant of Cerrado in the state of São Paulo, Brazil. Check List, Rio Claro, v. 10, n. 5, p. 1038-1043, 2014.

ARID, F. M.; CASTRO, P. R. M.; BARCHA, S. F. Estudos hidrogeológicos no município de São José do Rio Preto, SP. Boletim da Sociedade Brasileira de Geologia, São Paulo, v. 19, p. 43-69, 1970.

BARCHA, S. F.; ARID, F. M. Estudo da evapotranspiração na região norte-ocidental do estado de São Paulo. Revista de Ciências da Faculdade de Ciências e Letras, São Paulo, v. 1, p. 94-122, 1971.

BRESSAN, P. M.; KIERULFF, M. C. M.; SUGIEDA, A. M. Fauna ameaçada de extinção no estado de São Paulo: vertebrados. São Paulo: Fundação Parque Zoológico de São Paulo: Secretaria do Meio Ambiente, 2009. 645 p.

CAMPBELL, H. W.; CHRISTMAN, S. P. Field techniques for herpetofaunal community analysis. In: SCOTT-JR, N. J. (Ed.). Herpetological communities: a Symposium of the Society for the Study of Amphibians and Reptiles and the Herpetologist's League. Washington: U. S. Fish Wildlife Service, 1982. p. 193-200.

CECHIN, S. Z.; MARTINS, M. Eficiência de armadilhas de queda (pitfall traps) em amostragens de anfíbios e répteis no Brasil. Revista Brasileira de Zoologia, Curitiba, v. 17, n. 3, p. 729-740, 2000.

CICCHI, P. J. P.; SERAFIM, H.; SENA, M. A.; CENTENO, F. C.; JIM, J. Herpetofauna em uma área de Floresta Atlântica na Ilha Anchieta, município de Ubatuba, sudeste do Brasil. Biota Neotropica, Campinas, v. 9, n. 2, p. 201-212, 2009.

CONDEZ, T. H.; SAWAYA, R. J.; DIXO, M. Herpetofauna dos remanescentes de Mata Atlântica da região de Tapiraí e Piedade, SP, sudeste do Brasil. Biota Neotropica, Campinas, v. 9, n. 1, p. 157-185, 2009

FERRAREZZI, H. Sistemática filogenética de Elapomorphus, Phalotris e Apostolepis (Serpentes: Colubridae: Xenodontinae). 1993. 276 f. Dissertação (Mestrado em Ciências Biológicas) Universidade de São Paulo, São Paulo. 1993. 
FORLANI, M. C.; BERNARDO, P. H.; HADDAD, C. B. F.; ZAHER, H. Herpetofauna do Parque Estadual Carlos Botelho, São Paulo, Brasil. Biota Neotropica, Campinas, v. 10, n. 3, p. 265-308, 2010

GREENBERG, C. H.; NEARY, D. G.; HARRIS, L. D. A comparison of herpetofaunal sampling effectiveness of pitfall, single-ended, and double-ended funnel traps used with drift fences. Journal of Herpetology, Salt Lake City, v. 28, n. 3, p. 319-324, 1994.

HAMMER, Ø., HARPER, D. T. A.; RYAN, P. D. Paleontological statistics software package for education and data analysis. Paleontologia Electronica, Oslo, v. 4, n. 1, p. 1-9, 2001.

IUCN. List of threatened species. Version 2015-4. 2016. Disponível em: <http://www.iucnredlist.org $>$. Acesso em: 23 maio 2016

KRONKA, F. J. N.; MATSUKUMA, C. K.; NALON, M. A.; DELCALI, I. H.; ROSSI, M.; MATTOS, I. F. A.; SHINIKE, M. S.; PONTINHAS, A. A. S. Inventário florestal do estado de São Paulo. São Paulo: Instituto Florestal, 1993. 199 p.

MAGURRAN, A. E. Measuring biological diversity. Oxford: Blackwell Science, 2004. 256 p.

MARTINS, M.; OLIVEIRA, M. E. Natural history of snakes in forests of the Manaus region, Central Amazonia, Brazil. Herpetological Natural History, Victorville, v. 6, p. 78-150, 1998. MMA - MINISTÉRIO DO MEIO AMBIENTE. Portaria no 444, de 17 de dezembro de 2014. Reconhece como espécies da fauna brasileira ameaçadas de extinção aquelas constantes da "Lista Nacional Oficial de Espécies da Fauna Ameaçadas de Extinção". Ministério do Meio Ambiente, 2014.

NOGUEIRA, C. Ecologia histórica de Bothrops spp. (Serpentes: Viperidae: Crotalinae) simpátricas no Cerrado. 2001. $69 \mathrm{f}$. Dissertação (Mestrado em Ecologia) - Universidade de São Paulo, São Paulo. 2001.

NECCHI-JUNIOR, O. (Ed.). Fauna e flora dos fragmentos florestais remanescentes da região norte do estado de São Paulo. Ribeirão Preto: Holos, 2012. 301 p.

PEREIRA, D. N.; SAWAYA, R. J. Répteis Squamata de remanescentes florestais da região noroeste do estado de São Paulo. In: NECCHI-JUNIOR, O. (Ed.). Fauna e flora de fragmentos florestais remanescentes da região noroeste do estado de São Paulo. Ribeirão Preto: Holos, 2012. p. 227-241.
RECODER, R. S.; TEIXEIRA JUNIOR, M.; CAMACHO, A.; NUNES, P. M. S.; MOTT, T.; VALDUJO, P. H.; GHELLERE, J. M.; NOGUEIRA, C.; RODRIGUES, M. T. Reptiles of Serra Geral do Tocantins Ecological Station, Central Brazil. Biota Neotropica, Campinas, v. 11, n. 1, p. 263-281, 2011.

SAWAYA, R. J.; MARQUES, O. A. V.; MARTINS, M. Composição e história natural das serpentes de cerrado de Itirapina, São Paulo, sudeste do Brasil. Biota Neotropica, Campinas, v. 8, n. 2, p. $127-$ 149, 2008.

SBH - SOCIEDADE BRASILEIRA DE HERPETOLOGIA. Dispõe de informação sobre os anfíbios e répteis do Brasil. Disponível em: <http://www.sbherpetologia.org.br/>. Acesso em: 23 maio 2016

SCOTT-JR., N. J.; MAXWELL, T. C.; THORNTON-JR., O. W.; FITZGERALD, L. A.; FLURY, J. W. Distribution, habitat, and future of Harter's Water Snake, Nerodia harteri, in Texas. Journal of Herpetology, Salt Lake City, v. 23, n. 4, p. 373-389, 1989.

SPECIESLINK. Sistema distribuído de informação que integra em tempo real, dados primários de coleções científicas. 2016. Disponível em: <http://splink.cria.org.br/>. Acesso em: 25 maio 2016.

THOMÉ, M. T. C. Diversidade de anuros e lagartos em fisionomias de Cerrado na região de Itirapina, sudeste do Brasil. 2006. 52 f. Dissertação (Mestrado em Ecologia) - Universidade de São Paulo, São Paulo. 2006

UETANABARO, M.; SOUZA, F. L.; FILHO, P. L.; BEDA, A. F.; BRANDÃO, R. A. Anfíbios e répteis do Parque Nacional da Serra da Bodoquena, Mato Grosso do Sul, Brasil. Biota Neotropica, Campinas, v. 7, n. 3, p. 279-289, 2007.

ZAHER, H.; BARBO, F. E.; MARTÍNEZ, P. S.; NOGUEIRA, C.; RODRIGUES, M. T.; SAWAYA R. J. Répteis do estado de São Paulo: conhecimento atual e perspectivas. Biota Neotropica, Campinas, v. 11, n. 1a, p. 1-15, 2011. 